\title{
Site-Specific Fluorescence Polarization for Studying the Disaggregation of $\alpha$-Synuclein Fibrils by Small Molecules
}

\author{
Conor M. Haney, ${ }^{\dagger}$ Christina L. Cleveland, ${ }^{\dagger}$ Rebecca F. Wissner, ${ }^{\dagger}, \|$ Lily Owei, $^{\dagger}$ Jaclyn Robustelli, $^{\dagger}$ \\ Malcolm J. Daniels, ${ }^{\ddagger}$ Merve Canyurt, ${ }^{\dagger, \perp}$ Priscilla Rodriguez, ${ }^{\dagger}$ Harry Ischiropoulos, ${ }^{\S}$ Tobias Baumgart, ${ }^{\dagger}$ \\ and E. James Petersson*, ${ }^{\dagger}$ \\ ${ }^{\dagger}$ Department of Chemistry, University of Pennsylvania, 231 South 34th Street, Philadelphia, Pennsylvania 19104, United States \\ ${ }^{\ddagger}$ Pharmacology Graduate Group, University of Pennsylvania, 3400 Civic Center Boulevard, Philadelphia, Pennsylvania 19104, United \\ States \\ ${ }^{\S}$ Department of Pediatrics, Children’s Hospital of Philadelphia, Philadelphia, Pennsylvania 19104, United States
}

\section{Supporting Information}

ABSTRACT: Fibrillar aggregates of the protein $\alpha$-synuclein $(\alpha \mathrm{S})$ are one of the hallmarks of Parkinson's disease. Here, we show that measuring the fluorescence polarization (FP) of labels at several sites on $\alpha \mathrm{S}$ allows one to monitor changes in the local dynamics of the protein after binding to micelles or vesicles, and during fibril formation. Most significantly, these site-specific FP measurements provide insight into structural remodeling of $\alpha \mathrm{S}$ fibrils by small molecules and have the potential for use in moderate-throughput screens to identify small molecules that could be used to treat Parkinson's disease.

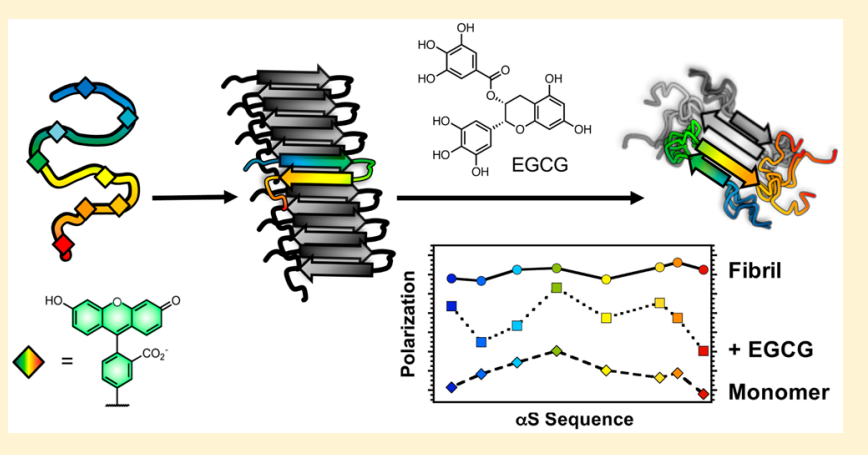

investigated as potential therapeutics to combat the progression of PD..$^{21-27}$ Although amyloid binding dyes such as ThT and $\mathrm{CR}$ are used in high-throughput screening to identify modifiers of aggregation, these dyes cannot be used to reliably detect morphological changes of $\alpha \mathrm{S}$ induced by small molecules, as it is likely that the dyes and candidate compounds compete for similar binding sites. ${ }^{28,29}$ Moreover, the optical properties of amyloid binding dyes may be affected by the presence of other compounds in solution. Indeed, our own analysis of small molecule remodeling experiments using ThT shows that the change in ThT fluorescence does not accurately reflect the change in the amount of insoluble $\alpha \mathrm{S}$ aggregates following small molecule treatment (see Figures S17, S25, and S26 and discussion below). Improved methods for monitoring the aggregation and disaggregation of $\alpha \mathrm{S}$ that yield meaningful structural information and are not susceptible to these drawbacks would greatly facilitate our understanding of these crucial processes.

Fluorescence polarization (FP) spectroscopy is a powerful technique for monitoring changes in the conformational mobility of fluorescently labeled molecules. ${ }^{30}$ By incorporating a fluorescent label into a protein, one can use FP to obtain a range of dynamic information. In 2007, the Lee laboratory demonstrated that FP can be used to monitor the aggregation of $\alpha \mathrm{S}^{31}$ In this study, the authors prepared nonspecifically

Received: October 14, 2016

Published: November 11, 2016 
labeled $\alpha \mathrm{S}$ by treating the recombinant protein with an aminereactive dye. Over the course of aggregation, FP values of the labeled protein increased in a manner that was consistent with a nucleation-dependent process characteristic of $\alpha \mathrm{S}$ assembly. ${ }^{32}$ Notably, the observed increase in polarization preceded fibril detection by amyloid binding dyes, suggesting that FP can be used to detect the formation of early oligomeric intermediates. Although this initial report demonstrated the utility of FP for monitoring $\alpha \mathrm{S}$ aggregation, the proteins used in these studies consisted of heterogeneous mixtures of labeled constructs. Therefore, we sought to determine whether site-specifically labeled $\alpha \mathrm{S}$ could be used in conjunction with FP to distinguish local changes in conformational freedom that occur during misfolding and aggregation of $\alpha \mathrm{S}$. Although several small molecules have been reported to remodel and/or disaggregate preformed $\alpha \mathrm{S}$ fibrils, robust, moderate- to high-throughput methods for examining this process in real time are currently lacking. Here, we show that we can use FP to monitor the conformational changes within $\alpha \mathrm{S}$ fibrils induced by small polyphenolic compounds and demonstrate that site-specific labels yield insight into their interaction with $\alpha \mathrm{S}$ fibrils and subsequent local conformational changes (Table 1).

\section{EXPERIMENTAL PROCEDURES}

Fluorescence Polarization (FP) Measurements. The concentrations of high-performance liquid chromatography (HPLC)-purified fluorescein maleimide (Fam)-labeled proteins were determined by UV-vis absorbance using a molar extinction coefficient of $68000 \mathrm{M}^{-1} \mathrm{~cm}^{-1}$ at $494 \mathrm{~nm}$. The concentration of wild-type (WT) $\alpha \mathrm{S}$ was also determined using UV-vis absorbance using a molar extinction coefficient of 5120 $\mathrm{M}^{-1} \mathrm{~cm}^{-1}$ at $280 \mathrm{~nm}$. Prior to FP measurements, the Famlabeled mutants were diluted into a solution of WT $\alpha \mathrm{S}$ in a 1:99 molar ratio to achieve a final total protein concentration of 100 $\mu \mathrm{M} ; 100 \mu \mathrm{L}$ portions of $10 \mu \mathrm{M}$ samples were prepared in triplicate by diluting the $100 \mu \mathrm{M}$ stock into $\alpha \mathrm{S}$ buffer $[20 \mathrm{mM}$ Tris and $100 \mathrm{mM} \mathrm{NaCl}(\mathrm{pH} \mathrm{7.5)].} \mathrm{Each} \mathrm{sample} \mathrm{was} \mathrm{then}$ gently vortexed and pipetted into an untreated Corning Costar black, clear flat bottom nonsterile 96-well plate (part no. 07200-567). All FP measurements here and below were taken using a Tecan (Mannedorf, Switzerland) Infinite F200 Pro microplate reader equipped with excitation $(485 \pm 20 \mathrm{~nm})$ and emission $(535 \pm 25 \mathrm{~nm})$ filters for Fam fluorescence.

Fluorescence Polarization Measurements in Sodium Dodecyl Sulfate (SDS). To measure the FP values of each mutant in the presence of SDS, three $20 \mu \mathrm{L}$ replicates were prepared by dilution of $\alpha \mathrm{S}-\mathrm{C}^{\mathrm{Fam}}{ }_{\mathrm{X}}$ protein stock solutions (concentration determined as described above) with a $25 \mathrm{mM}$ SDS stock in $\alpha \mathrm{S}$ buffer and $\alpha \mathrm{S}$ buffer to yield samples comprised of $1 \mu \mathrm{M} \alpha \mathrm{S}-\mathrm{C}^{\mathrm{Fam}}{ }_{X}$ in $10 \mathrm{mM}$ SDS, $20 \mathrm{mM}$ Tris, and $100 \mathrm{mM} \mathrm{NaCl}$ ( $\mathrm{pH} 7.5$ ). The samples were gently vortexed and pipetted into an untreated Grenier black, clear flat bottom nonsterile 384-well plate (part no. 781209) and assessed on the Tecan F200 Pro microplate reader after incubation at room temperature for $5 \mathrm{~min}$.

FP Measurements in Large Unilamellar Vesicles (LUVs). LUVs were prepared using 100\% 1,2-dioleoyl-snglycero-3-phospho-L-serine (DOPS). Lipids were vacuum-dried to form a lipid film and rehydrated with $150 \mathrm{mM} \mathrm{NaCl}, 20 \mathrm{mM}$ HEPES, and $1 \mathrm{mM}$ TCEP ( $\mathrm{pH}$ 7.4). Liposome solutions were sonicated for $20 \mathrm{~min}$ and extruded through $200 \mathrm{~nm}$ Whatman nuclepore membranes (GE Life Sciences, Pittsburtgh, PA) 15 times. Liposome solutions were stored at $4{ }^{\circ} \mathrm{C}$. To measure the
FP values of each mutant in the presence of DOPS LUVs, three $20 \mu \mathrm{L}$ replicates were prepared in bulk at $300 \mathrm{nM} \alpha \mathrm{S}-\mathrm{C}^{\text {Fam }}{ }_{X}$ and $1.5 \mathrm{mM} 200 \mathrm{~nm}$ DOPS LUVs (concentration of total DOPS) in $20 \mathrm{mM}$ HEPES, $150 \mathrm{mM} \mathrm{NaCl}, \mathrm{pH} 7.0$ buffer (vesicle buffer). The samples were gently vortexed and pipetted into an untreated Grenier black, clear flat bottom nonsterile 384-well plate and assessed on the Tecan F200 Pro microplate reader after incubation at room temperature for $5 \mathrm{~min}$.

Fluorescence Microscopy Imaging of $\alpha \mathrm{S}-\mathrm{C}^{\mathrm{Fam}}{ }_{x}$ Bound to Giant Unilamellar Vesicles (GUVs). GUVs composed of 99.7\% DOPS and 0.3\% Texas red-1,2-dihexadecanoyl-snglycero-3-phosphoethanolamine triethylammonium salt (Texas Red-DHPE) were prepared by electroformation in a $300 \mathrm{mM}$ sucrose solution as previously described. ${ }^{33}$ An imaging chamber was formed between two coverslips $(25 \mathrm{~mm} \times 25$ $\mathrm{mm}$ ). The chamber had a total volume of $36 \mu \mathrm{L}$ and was filled by dilution of a $6 \mu \mathrm{M}$ GUV dispersion into buffer, yielding a solution with final concentrations of $200 \mathrm{mM}$ sucrose, $33 \mathrm{mM}$ $\mathrm{NaCl}, 7 \mathrm{mM}$ HEPES, $0.3 \mathrm{mM}$ TCEP, and $\sim 0.3-0.5 \mu \mathrm{M}$ total $\alpha \mathrm{S}-\mathrm{C}_{X}^{\text {Fam }}{ }_{X}(\mathrm{pH} 7)$. The protein- and GUV-containing solution was incubated for at least $10 \mathrm{~min}$ before being imaged with a confocal fluorescence microscope using a $60 \times, 1.1$ NA objective lens (Olympus, Center Valley, PA). The incubation and imaging processes were performed at room temperature $(20$ $\left.{ }^{\circ} \mathrm{C}\right)$.

Aggregation Assays. Aggregation reactions were performed by diluting each labeled construct into a mixture of WT $\alpha \mathrm{S}$ in a 1:99 molar ratio to a final concentration of $100 \mu \mathrm{M}$. Aggregation samples were prepared in triplicate. Aggregation was initiated by shaking the solution at $37^{\circ} \mathrm{C}$ and $1500 \mathrm{rpm}$ on an IKA MS3 digital orbital shaker in parafilm-sealed $1.7 \mathrm{~mL}$ Eppendorf tubes to ensure minimal solvent evaporation. At each time point, aliquots were removed from the aggregation reaction mixture and assessed by $\mathrm{FP}$ and $\mathrm{CR}$ absorbance in separate assays. Fluorescence polarization measurements were obtained by dilution of $10 \mu \mathrm{L}$ of the aggregation solution into $90 \mu \mathrm{L}$ of $\alpha \mathrm{S}$ buffer (100 $\mu \mathrm{L}$ total volume). The samples were gently vortexed and transferred to an untreated Corning Costar black, clear flat bottom nonsterile 96-well plate and analyzed on a Tecan F200 plate reader. CR absorbance measurements were taken by dilution of $10 \mu \mathrm{L}$ of the aggregation solution into 140 $\mu \mathrm{L}$ of $20 \mu \mathrm{M}$ CR dissolved in $\alpha \mathrm{S}$ buffer. The samples were allowed to sit at room temperature for $15 \mathrm{~min}$ prior to being transferred to an untreated Corning Costar black, clear flat bottom nonsterile 96-well plate and absorbance measurements acquired on a Tecan M1000 plate reader using a wavelength range of 230-700 nm. Following completion of the aggregation assay, the samples were split into two separate aliquots; fibrils in each aliquot were then pelleted by centrifugation ( 13200 rpm for $90 \mathrm{~min}$ at $4{ }^{\circ} \mathrm{C}$ ), and the supernatant was removed. One aliquot was immediately resuspended in an equal volume (relative to supernatant) of $\alpha \mathrm{S}$ buffer, and the measurements described above were repeated on the resuspended fibril sample. The second aliquot was frozen at $-20^{\circ} \mathrm{C}$ until it was analyzed by transmission electron microscopy (TEM) as described below.

Small Molecule Remodeling Assays. The 1\% Famlabeled $\alpha \mathrm{S}$ fibrils of each labeled mutant were prepared for small molecule remodeling assays as described above. Stock solutions of dopamine, epigallocatechin gallate (EGCG), and nordihydroguiaretic acid (NDGA) were prepared by mass-tovolume ratio at $1 \mathrm{mM}$ in $\alpha \mathrm{S}$ buffer; NDGA was solubilized in $20 \%$ EtOH. Small molecule stock solutions at $500 \mu \mathrm{M}$ 
dopamine, $500 \mu \mathrm{M}$ EGCG, and $500 \mu \mathrm{M}$ NDGA were prepared by 2 -fold dilution of the $1 \mathrm{mM}$ stocks in $\alpha \mathrm{S}$ buffer; all stocks were prepared immediately prior to the performance of each remodeling assay. The final concentration of ethanol in the NDGA-treated fibrils was $2 \%$ by volume. The remodeling assay was first tested in triplicate by treating $10 \mu \mathrm{M} \alpha \mathrm{S}$ fibrils with $100 \mu \mathrm{M}$ freshly prepared dopamine, EGCG, and NDGA; as a control, buffer was added to the fibrils in lieu of small molecules. Controls were prepared by diluting $30 \mu \mathrm{L}$ of the resuspended $1 \% \alpha \mathrm{S}-\mathrm{C}^{\mathrm{Fam}}{ }_{X}$ fibrils in $270 \mu \mathrm{L}$ of $\alpha \mathrm{S}$ buffer, gently vortexing, and splitting the sample into three wells, $100 \mu \mathrm{L}$ per well, of an untreated Corning Costar black, clear flat bottom nonsterile 96-well plate. The fibril solutions to be treated with the small molecules were prepared by diluting $90 \mu \mathrm{L}$ of the resuspended fibrils in $630 \mu \mathrm{L}$ of $\alpha \mathrm{S}$ buffer, gently vortexing, and splitting the sample into nine wells, $80 \mu \mathrm{L}$ per well of the same plate as described above. Prior to the addition of the small molecule solution to the $\alpha \mathrm{S}$ fibrils, the FP for all the wells was measured and the gain optimized. Small molecules were added from freshly prepared $500 \mu \mathrm{M}$ small molecule stock solutions in $\alpha \mathrm{S}$ buffer (20 $\mu \mathrm{L} /$ well, in triplicate) to start the assay. Over the course of $3 \mathrm{~h}$, the instrument performed the following actions every $1.5 \mathrm{~min}$ : orbital shaking for $2 \mathrm{~s}$ with an amplitude of 1 $\mathrm{mm}$ followed by an FP measurement of all wells using the gain optimized prior to the small molecule addition. All measurements were taken at room temperature.

FP of Soluble and Insoluble Fractions after the Small Molecule Remodeling Assay. At the end of the remodeling assay (i.e., $3 \mathrm{~h}$ post-small molecule addition), the three replicates from each condition (control, dopamine, EGCG, and NDGA) were removed from the 96-well plate, combined, and gently vortexed. After being vortexed, the combined samples were split into four Eppendorf tubes at $60 \mu \mathrm{L}$ per tube. All of the samples were then pelleted by centrifugation ( 13200 rpm for $90 \mathrm{~min}$ at $4{ }^{\circ} \mathrm{C}$ ) to separate the soluble and insoluble fractions. Because it is difficult to completely remove all of the supernatant without disturbing the pellet for small samples, the following procedure was used. After centrifugation, the following volumes of supernatant (soluble fraction) were removed from each of the four tubes: $0,10,20$, and $30 \mu \mathrm{L}$. The 10, 20, and $30 \mu \mathrm{L}$ portions of the supernatant that were removed were combined, gently vortexed, and transferred to an untreated Grenier black, clear flat bottom nonsterile 384-well plate to be measured as the soluble fraction for the respective positions and conditions. The removed supernatant was replaced with the same volume of buffer containing the respective small molecules. The small molecule solutions used to replace the supernatant were prepared from the small molecule stocks made at the start of the remodeling assay, diluted to the same concentration as that used in the assay, and centrifuged $\left(13200 \mathrm{rpm}\right.$ for $10 \mathrm{~min}$ at $\left.4{ }^{\circ} \mathrm{C}\right)$. All four pellets per condition (the $-0,-10,-20$, and $-30 \mu \mathrm{L}$ soluble fraction pellets) were then resuspended by vortexing at high speed. The resuspended pellets were then transferred to an untreated Grenier black, clear flat bottom nonsterile 384-well plate (split into three wells per Eppendorf tube, $20 \mu \mathrm{L}$ per well) to be measured as the insoluble fractions. The FP measurememts of these samples were used to extrapolate a FP measurement for the corresponding insoluble material as described in the Supporting Information.

Transmission Electron Microscopy (TEM). TEM was performed on an FEI Tecnai T12 instrument with an accelerating voltage of $120 \mathrm{kV}$. Fibril samples obtained from aggregation and centrifugation and stored at $-20{ }^{\circ} \mathrm{C}$ as dry pellets were resuspended in $20 \mathrm{mM}$ Tris and $100 \mathrm{mM} \mathrm{NaCl}$ ( $\mathrm{pH}$ 7.5). Glow-discharged carbon Formvar-coated 300-mesh $\mathrm{Cu}$ grids were inverted over a $10 \mu \mathrm{L}$ drop of sample and allowed to rest for $2 \mathrm{~min}$ at room temperature. After this time, the excess solution was wicked off and the grid was washed for $2 \times 10 \mathrm{~s}$ with water, and the grids were stained for $3 \times 15 \mathrm{~s}$ with $2 \%(\mathrm{w} / \mathrm{v})$ ammonium molybdate $(\mathrm{pH} 7.8)$ in water. The grids dried at room temperature for $2 \mathrm{~min}$ and then were imaged. Images were collected at magnifications ranging from $11000 \times$ to $42000 \times$. TEM image analysis was performed manually using Gatan Digital Micrograph software (Gatan, Inc., Pleasanton, $\mathrm{CA}$ ). Fibril length and width analysis is described in the Supporting Information.

Small Molecule Remodeling TEM. Fibril samples comprised of $\alpha \mathrm{S} \mathrm{WT}, 1 \% \alpha \mathrm{S}-\mathrm{C}^{\mathrm{Fam}}{ }_{9}$ in $99 \% \alpha \mathrm{S} \mathrm{WT}$, or $1 \%$ $\alpha \mathrm{S}-\mathrm{C}^{\mathrm{Fam}}{ }_{114}$ in $99 \% \alpha \mathrm{S}$ WT $(10 \mu \mathrm{M}$ fibril based on the monomeric total $\alpha \mathrm{S}$ concentration) were treated with dopamine, EGCG, or NDGA $(100 \mu \mathrm{M})$ in triplicate as described above. Three hours after small molecule treatment under plate reader-based kinetic assay conditions described above, a representative single sample was removed from the plate and used to prepare carbon Formvar-coated 300-mesh $\mathrm{Cu}$ grids for negative-stain TEM as described above. Additional images of these samples are shown in the Supporting Information.

\section{RESULTS AND DISCUSSION}

Recently, we reported the use of inteins as purification tags for the efficient production of labeled $\alpha \mathrm{S}$ to study conformational changes using Förster resonant energy transfer (FRET) and cellular trafficking of fibrils. ${ }^{34}$ To produce a library of sitespecifically labeled $\alpha \mathrm{S}$ for FP studies, we expressed constructs as $\mathrm{His}_{6}$-tagged Mxe GyrA intein fusions containing single Cys mutations (Figure 1). Following isolation of the intein-tagged proteins from the cell lysate by Ni-NTA chromatography, each construct was labeled using an excess of fluorescein maleimide (Fam) for $3-5 \mathrm{~h}$ at $37^{\circ} \mathrm{C}$. After labeling, each construct was further purified by ion-exchange chromatography and HPLC. HPLC-purified proteins were characterized by matrix-assisted laser desorption ionization mass spectrometry (MALDI-MS) and polyacrylamide gel electrophoresis (PAGE) analysis. MALDI-MS of trypsin digestion fragments confirmed that the labeling of $\alpha \mathrm{S}$ proceeded in a specific and quantitative manner (see Figures S4-S7).

We began our studies by measuring the polarization values associated with each labeled protein in detergent-free buffer. To avoid intermolecular fluorophore interactions (such as homoFRET, which may lead to a decrease in polarization ${ }^{35}$ ), labeled $\alpha \mathrm{S}$ was diluted into a solution of WT $\alpha \mathrm{S}$ in a 1:99 molar ratio for most FP measurements [micelle and vesicle binding experiments used $100 \%$ labeled $\alpha \mathrm{S}$ (see the Supporting Information for details)]. Because $\alpha \mathrm{S}$ is intrinsically disordered, we expected that FP values corresponding to monomeric $\alpha \mathrm{S}$ would be similar for each labeled mutant. Intriguingly, we found that the polarization of the Fam label was dependent on its location within the $\alpha \mathrm{S}$ sequence. These results are consistent with both in vitro and in cell NMR studies, suggesting that the residues in the C-terminal tail (96-140) exhibit a degree of conformational freedom greater than that of the residues residing in the N-terminus $(1-60)$ and the so-called nonamyloid-containing (NAC, 61-95) domain., ${ }^{6,36}$ 


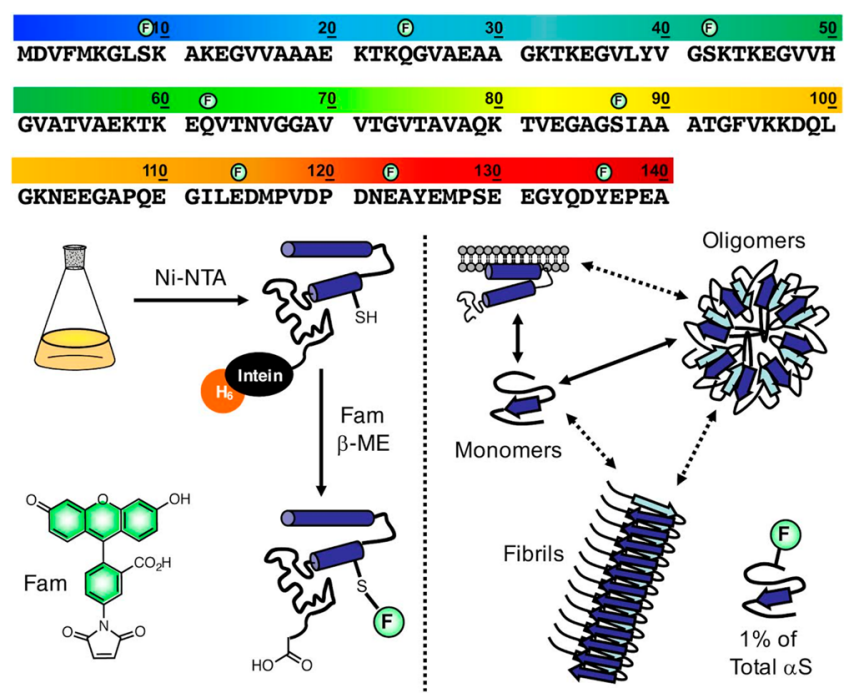

Figure 1. Labeling $\alpha$-synuclein $(\alpha \mathrm{S})$ for studies of aggregation. Protein sequence of $\alpha \mathrm{S}$ (top). Green circles indicate positions for fluorophore attachment. Cys mutants of $\alpha \mathrm{S}$ are purified using a C-terminal intein tag (bottom left), treated with $\beta$-mercaptoethanol $(\beta$-ME) to cleave the intein, and labeled with fluorescein maleimide (Fam). Monomers of $\alpha \mathrm{S}$ can form helices when bound to membranes (bottom right). Disordered monomers can misfold and aggregate to form oligomers or fibrils. Aggregation experiments were performed with $1 \%$ labeled protein to prevent fluorophore-fluorophore interactions.

To further demonstrate that site-specific labels yield information about local $\alpha \mathrm{S}$ dynamics, we took FP measurements of each $\alpha \mathrm{S}$ mutant bound to sodium dodecyl sulfate (SDS) micelles or large unilamellar vesicles (LUVs). Previous NMR and single-molecule fluorescence studies have shown that upon binding to SDS, the first 98 residues of $\alpha \mathrm{S}$ fold into a pair of curved antiparallel helices that are connected by a short turn region spanning residues $38-44^{37,38}$ The helix-turn-helix motif is followed by a predominantly disordered C-terminal tail consisting of residues $98-140 .{ }^{37}$ However, in the presence of LUVs, residues $1-98$ of $\alpha \mathrm{S}$ have been shown to adopt an extended helical conformation by smFRET. ${ }^{39}$ In the presence of SDS and 1,2-dioleoyl-sn-glycero-3-phospho-L-serine (DOPS) LUVs, constructs with Fam labels at position 9, 24, 42, 62, or 87 show increased FP, while constructs with Fam labels at position 114, 123, or 136 show decreased FP (Figure 2 and Figures S9 and S10). The increase in FP observed in the presence of SDS versus DOPS LUVs for labels at position 42, 62 , or 87 is consistent with a rearrangement from the micellebound to vesicle-bound helical structures. Circular dichroism (CD) spectroscopy measurements confirm that each labeled construct adopts an $\alpha$-helical conformation in the presence of SDS (Figure S8a,b). We also wished to image the bound $\alpha \mathrm{S}$ constructs, because some evidence exists for remodeling of liposomes by $\alpha \mathrm{S}$ to form cylindrical micelles. ${ }^{40}$ While SDS micelles and LUVs are too small for imaging, microscopy images of DOPS giant unilamellar vesicles (GUVs) confirm that our labeled $\alpha \mathrm{S}$ constructs bind to the surfaces of vesicles.

Our FP data are consistent with the existing NMR and electron paramagnetic resonance (EPR) structures of $\alpha \mathrm{S}$ bound to SDS micelles and previous studies of the $\alpha \mathrm{S}$ conformation bound to LUVs. ${ }^{9,37,39,41}$ They are also consistent with the previous observation that monomeric, disordered $\alpha \mathrm{S}$ has longrange contacts between the $\mathrm{N}$ - and C-termini that are released upon binding to micelles or vesicles ${ }^{42,43}$ (decreasing FP values
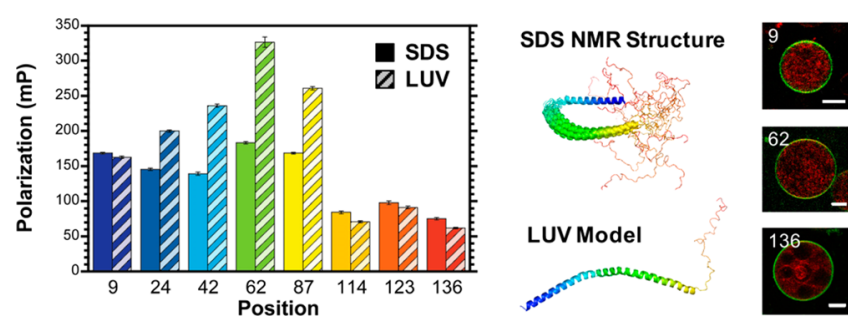

Figure 2. In the left panel, fluorescence polarization (FP) reveals local dynamics in proteins bound to micelles or vesicles. FP measurements of $\alpha \mathrm{S}$ constructs labeled with Fam at the noted positions, bound to sodium dodecyl sulfate (SDS) micelles or large unilamellar vesicles (LUVs) composed of 1,2-dioleoyl-sn-glycero-3-phospho-L-serine (DOPS). In the middle panel, the SDS-bound structure of $\alpha \mathrm{S}$ (PDB entry 1XQ8) and a model of the LUV-bound $\alpha \mathrm{S}$ structure, colored to match the sequence diagram in Figure 1, are shown for comparison to the FP data. ${ }^{37,39}$ The right panels are images of Fam-labeled $\alpha \mathrm{S}$ constructs bound to giant unilamellar vesicles (GUVs) composed of DOPS and Texas Red-1,2-dihexadecanoyl-sn-glycero-3-phosphoethanolamine. Numbers indicate the position of the Fam label on $\alpha \mathrm{S}$ constructs. The scale bar is $10 \mu \mathrm{m}$.

for C-terminal positions compared to monomer in buffer). Importantly, these experiments show that despite $\alpha \mathrm{S}$ being bound to large, slowly rotating micelles or LUVs, the FP measurements reflect the local dynamics of the protein around the site of attachment of the dye to $\alpha \mathrm{S}$.

Next, we used FP to monitor the assembly of $\alpha \mathrm{S}$ into amyloid fibrils. Aggregation reactions were performed by diluting each labeled construct into a mixture of WT $\alpha \mathrm{S}$ in a 1:99 molar ratio and agitating the solution at $1500 \mathrm{rpm}$ and 37 ${ }^{\circ} \mathrm{C}$. Periodically, separate aliquots of the aggregation reaction mixture were removed for FP and CR analysis. CR binding assays demonstrated that the kinetic profiles of aggregation assays containing $1 \%$ labeled $\alpha \mathrm{S}$ are very similar to those obtained using WT alone. Example data for $\alpha \mathrm{S}-\mathrm{C}^{\mathrm{Fam}}{ }_{9}$ are shown in Figure 3; data for all other constructs are included in the Supporting Information (Figure S12; Tables S3 and S4 detail forward aggregation kinetics for each construct).

Both FP and CR measurements generated sigmoidal curves consistent with a nucleation-dependent mechanism of amyloid aggregation. However, the kinetic profiles obtained using FP varied on the basis of the position of the incorporated label. The $t_{1 / 2}$ for the changes in FP varied from 6.7 to $27 \mathrm{~h}$. This observation is consistent with some labeling positions leading to retarded co-incorporation of the labeled protein with the wild type (WT); several other explanations are plausible. For example, it is also possible that these positions indicate differences in local conformational restriction around the labeled site in a time-dependent manner during aggregation, or that addition of labeled protein perturbs the aggregation pathway. While we interpret the change in $t_{1 / 2}$ by FP relative to $\mathrm{CR}$ as being indicative of slower co-incorporation of labeled protein, further investigation of the mechanism of aggregation as reported by FP is underway. Regardless, aggregation end point assays showed that even constructs exhibiting a slower increase in FP, such as $\alpha \mathrm{S}-\mathrm{C}^{\mathrm{Fam}}{ }_{42}$, were ultimately incorporated well into the fibrils. We determined the percent of incorporation of labeled $\alpha \mathrm{S}$ by gel imaging and the morphology of the fibrils by TEM imaging (Figures S14, S15, S29, and S30; these include larger versions of the TEM images shown in Figure 3 ). These kinetic and end point assays show that our chosen labeling positions were not highly disruptive to 

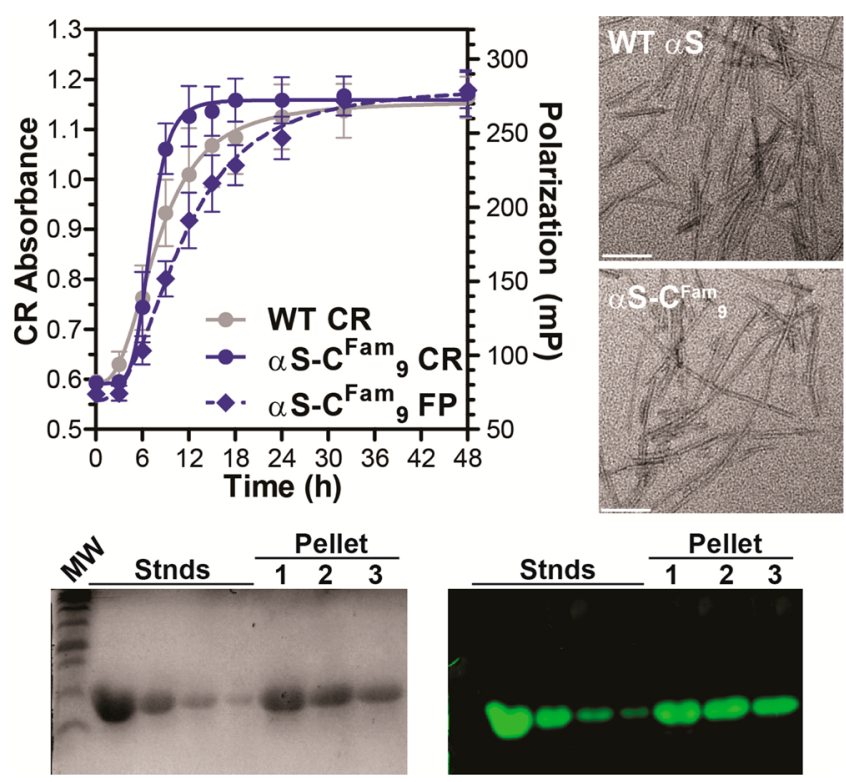

Figure 3. Monitoring forward aggregation and validating labeled constructs. The top left panel shows the aggregation kinetics of fibrils made with WT $\alpha \mathrm{S}$ or $1 \% \alpha \mathrm{S}-\mathrm{C}^{\mathrm{Fam}}{ }_{9}$ monitored by the Congo Red (CR) absorbance ratio $(540 \mathrm{~nm} / 480 \mathrm{~nm})$ as well as changes in FP. The top right panels are transmission electron microscopy (TEM) images showing that fibrils made with $1 \% \alpha \mathrm{S}-\mathrm{C}^{\mathrm{Fam}}{ }_{9}$ are morphologically similar to WT $\alpha$ S fibrils. The scale bar is $100 \mathrm{~nm}$. In the bottom panels, gel analysis demonstrates that $\alpha \mathrm{S}-\mathrm{C}^{\mathrm{Fam}}{ }_{9}$ is incorporated into fibrils stoichiometrically. Standards (Stnds) of $1 \% \alpha \mathrm{S}-\mathrm{C}^{\mathrm{Fam}}{ }_{9}$ loaded at $100,50,25$, and $12.5 \mu \mathrm{M}$. Molecular weight (MW) markers at 11, 17, $22,25,32,46$, and $58 \mathrm{kDa}$. The same battery of experiments was conducted on $\alpha \mathrm{S}$ constructs labeled at Cys24, -42, -62, -87, -114, -123, or -136 . These data are shown in the Supporting Information.

incorporation of $\alpha \mathrm{S}$ into fibrils or the resulting supramolecular structure (see Table 1 .

For those label positions ruled to be sufficiently nonperturbing, we examined the FP measurements of isolated fibrils. After $48 \mathrm{~h}$, fibrils were pelleted by centrifugation and resuspended in buffer, and FP was measured, showing only slight differences between positions, reflecting the expected high degree of conformational restriction within fibrils. However, even in these fibrils that are $>100 \mathrm{~nm}$ in length, the FP values are still significantly less than $470 \mathrm{mP}$, the limiting value for the polarization (anisotropy) of fluorescein. ${ }^{44}$ This means that even in fibrils, some dynamic range remains that allows us to interpret local differences in FP that reflect conformational dynamics in a site-dependent manner. For example, we can see that the C-terminal tail is more restricted for $\alpha \mathrm{S}$ molecules within fibrils than for SDS-bound $\alpha \mathrm{S}$. This is interesting in light of previous studies of $\alpha \mathrm{S}$ fibrils, particularly recent solid state NMR studies by Rienstra and co-workers. ${ }^{18}$ Although they do not observe crosspeaks allowing them to assign structures to the terminal regions of $\alpha \mathrm{S}$, our data suggest that these regions are conformationally restricted in fibrils. It should be noted that our aggregation conditions differ from theirs, and our measurements are taken on suspensions rather than packed fibrils for NMR; therefore, this may contribute to differences in our observations.

We also examined aggregation reactions near $t_{1 / 2}$ to see whether we could observe oligomers or other intermediates using FP. We pelleted the insoluble material after aggregation for 6,9 , or $12 \mathrm{~h}$ and measured the FP of the supernatant and of the pellet after resuspension (see Figure S13). We found that the FP readings of the supernatant remained similar to those of monomeric $\alpha \mathrm{S}$, suggesting that the remaining soluble species do not differ considerably from monomeric $\alpha \mathrm{S}$ with respect to fluorophore conformational freedom. In contrast, the FP readings of the pellet were intermediate between monomer and fibril levels and grew steadily during aggregation. This may reflect the presence of smaller, protofibril aggregates at 6,9, and $12 \mathrm{~h}$, or it may be due to difficulties in completely washing away the monomer from these very small pellets. Further analysis of aggregations at early time points using techniques such as atomic force microscopy (AFM) will be required to determine the nature of these intermediate FP signals.

\section{Table 1. Forward Aggregation Studies}

$\begin{array}{lclcr}\alpha \mathrm{S} & t_{1 / 2}{ }^{\mathrm{CR}}(\mathrm{h})^{a} & t_{1 / 2}{ }^{\mathrm{FP}}(\mathrm{h})^{a} & \text { fibril FP }(\mathrm{mP})^{b} & \text { width }(\mathrm{nm})^{c} \\ \mathrm{WT} & 7.5 \pm 0.4 & \mathrm{NA} & \mathrm{NA} & 9.9 \pm 0.9 \\ 9 & 7.0 \pm 0.1 & 11.1 \pm 0.5 & 312 \pm 4 & 9.9 \pm 0.7 \\ 24 & 6.9 \pm 0.1 & 12.6 \pm 0.4 & 307 \pm 7 & 9.9 \pm 0.7 \\ 42 & 7.6 \pm 0.3 & 27 \pm 8 & 330 \pm 7 & 10.0 \pm 0.7 \\ 62 & 10 \pm 1 & 17 \pm 1 & 333 \pm 6 & 9.9 \pm 0.7 \\ 87 & 10.9 \pm 0.7 & 15.3 \pm 0.9 & 310 \pm 5 & 10.1 \pm 0.7 \\ 114 & 10 \pm 2 & 9.4 \pm 0.4 & 335 \pm 3 & 10.0 \pm 0.5 \\ 123 & 8 \pm 1 & 6.7 \pm 0.2 & 345 \pm 5 & 10.0 \pm 0.8 \\ 136 & 7.5 \pm 0.4 & 6.9 \pm 0.4 & 330 \pm 4 & 9.8 \pm 0.7\end{array}$

${ }^{a}$ Fibril kinetic parameters determined by fits to aggregation curves shown in Figure S11a-c. ${ }^{b} \mathrm{FP}$ values for pelleted, resuspended fibrils determined as described in the Supporting Information. ${ }^{c}$ Fibril width determined from analysis of TEM images. Example images are shown in Figure S30a-c.

We next chose to examine whether we could use this technique to monitor the disaggregation of $\alpha \mathrm{S}$ fibrils. We began by using harsh conditions known to reliably dissolve fibrils, boiling in the presence of SDS. ${ }^{45} \alpha \mathrm{S}$ fibrils containing each labeled construct were agitated for $48 \mathrm{~h}$ and separated from the aggregation reaction mixture by centrifugation. The freshly resuspended fibrils were then treated with excess SDS, boiled for $15 \mathrm{~min}$, and allowed to cool to room temperature. Following the disaggregation procedure, the FP values uniformly decreased to those corresponding to the SDSbound conformation observed previously by direct addition of SDS to monomeric $\alpha \mathrm{S}$ (Figure S16). Sedimentation gel analyses confirmed that $\alpha \mathrm{S}$ fibrils were efficiently dissolved following treatment with SDS. These studies demonstrate that we can use FP to reliably monitor the dissolution of $\alpha \mathrm{S}$ fibrils without the addition of any exogenous small molecule probe such as ThT.

Several small molecules are known to remodel and/or disaggregate preformed $\alpha \mathrm{S}$ fibrils. ${ }^{21,22,26,27,46-51}$ In some cases, it has been shown that remodeled $\alpha \mathrm{S}$ is less toxic to cultured neurons. However, the molecular details underlying these structural changes and how they mitigate fibril toxicity are poorly understood. Dopaminergic nerve cell death is one of the pathological hallmarks of PD. ${ }^{1}$ Thus, several groups have examined the effect of dopamine and its oxidized derivatives on the assembly of $\alpha \mathrm{S}^{46,48,50-52}$ In $2004, \mathrm{Li}$ et al. ${ }^{21}$ reported that dopamine treatment led to the disaggregation of preformed $\alpha \mathrm{S}$ fibrils. Surprisingly, the dopamine-mediated disaggregation of $\alpha \mathrm{S}$ fibrils has not been described since this initial report.

In the following years, several aromatic compounds containing vicinal hydroxyl groups have been identified as 

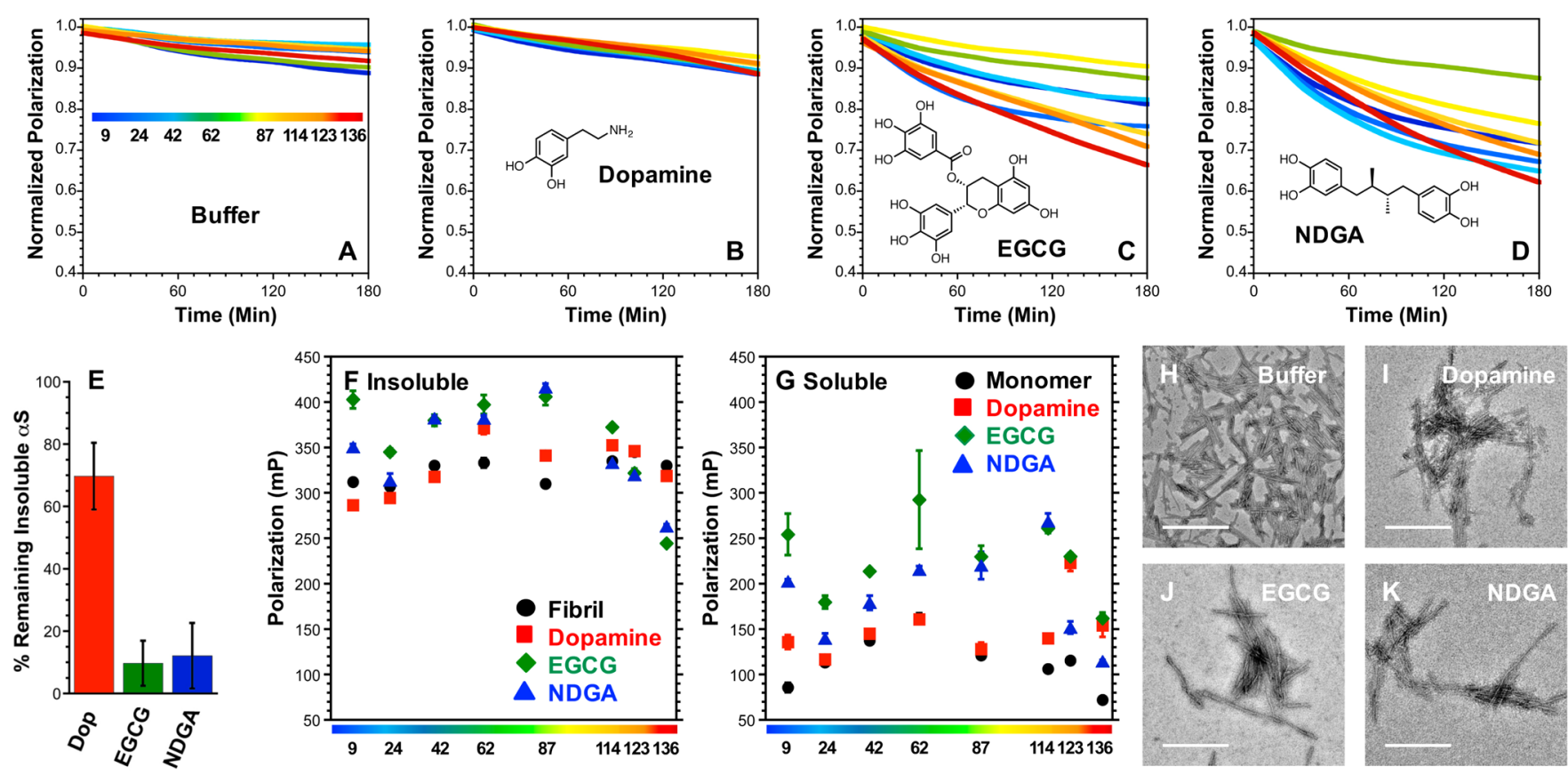

Figure 4. Monitoring fibril disaggregation by FP. (A-D) Fibrils made with $1 \%$ Fam-labeled $\alpha \mathrm{S}$ constructs (at position 9, 24, 42, 62, 87, 114, 123, or 136) were incubated with solutions of buffer, dopamine (Dop), epigallocatechin gallate (EGCG), or nordihydroguiaretic acid (NDGA). FP measurements are normalized to aid comparison of kinetics. Raw FP values are reported in the Supporting Information. (E) After incubation for $3 \mathrm{~h}$, the reaction mixtures were pelleted by centrifugation. A portion of the insoluble fraction was boiled in SDS and loaded onto a gel for quantification relative to buffer-treated fibrils, as described in the Supporting Information. (F and G) FP measurements of the resuspended insoluble and soluble fractions are shown. FP data for untreated fibrils and for monomer are shown for comparison. (H-K) TEM images of WT $\alpha \mathrm{S}$ fibrils, treated with the same disaggregation protocol, deposited on copper grids, and negatively stained with ammonium molybdate. The scale bar is $200 \mathrm{~nm}$. Larger TEM images are shown in Figure S31a-d.

efficient amyloid remodeling agents. Among the most wellstudied examples is epigallocatechin gallate (EGCG), a flavonoid that is found in high concentrations in green tea. In 2010, Bieschke et al. ${ }^{23}$ demonstrated that EGCG transforms $\alpha \mathrm{S}$ fibrils into smaller oligomeric species that are nontoxic to mammalian cells. Time-resolved TEM and AFM imaging revealed that EGCG converts $\alpha \mathrm{S}$ fibrils into poorly defined amorphous aggregates. $\mathrm{CD}$ spectroscopy experiments showed a progressive loss of $\beta$-sheet content in the presence of EGCG, demonstrating that binding of the compound led to a significant alteration of $\alpha \mathrm{S}$ secondary structure. ThT binding measurements were consistent with a decreasing amyloid content. However, because EGCG and ThT likely compete for similar binding sites, the reported remodeling kinetics may be unreliable and reveal no information about local conformational changes.

Having shown that we can use FP to monitor $\alpha \mathrm{S}$ folding, aggregation, and disaggregation, we reasoned that we could use this technique to gain insight into the mechanism by which small molecules remodel $\alpha \mathrm{S}$ fibrils. Here, we chose to examine the remodeling capacity of dopamine, EGCG, and an additional flavonoid, nordihydroguiaretic acid (NDGA). Previously, NDGA has been shown to inhibit $\alpha \mathrm{S}$ aggregation and to disassemble $\alpha \mathrm{S}$ oligomers that form in the presence of $\mathrm{FeCl}_{3}{ }^{27}$ However, prior to this work, the effect of NDGA treatment on the structure of $\alpha \mathrm{S}$ fibrils has not been described.

Fibrils containing each labeled mutant were treated with dopamine, EGCG, or NDGA and continuously monitored using FP (Figure 4A-D). Prolonged incubation with stoichiometric or excess amounts of dopamine had little effect on the observed FP values for any of the labeled mutants when compared to those from control experiments with buffer alone. These data are consistent with TEM images of WT $\alpha$ S samples, which do not show significant changes in the morphology of fibrils from dopamine-treated samples as compared to those treated with buffer only (Figure $4 \mathrm{H}-\mathrm{K}$ and Supporting Information). In contrast, rapid changes in FP for several labeled constructs were seen upon addition of EGCG or NDGA. For EGCG, larger changes in FP occurred for labels at C-terminal residues $(114,123$, and 136), consistent with earlier reports suggesting that EGCG preferentially interacts with the C-terminus of $\alpha \mathrm{S}$ in oligomers. ${ }^{47,49}$ Remarkably, treatment with excess NDGA produced a substantial change in FP for labels at every position except 62 . These proteinwide changes may be indicative of the binding of multiple molecules of NGDA per $\alpha \mathrm{S}$ molecule, resulting in dramatic remodeling of $\alpha \mathrm{S}$ fibrils. Indeed, the fact that we observe quenching of Fam at many locations upon addition of EGCG or NDGA supports this idea. Control experiments demonstrating that the observed effects on FP are not simply the result of fluorescence quenching but must result from changes in local protein dynamics are detailed in the Supporting Information. In all cases, the fibrils were treated with a 10 -fold excess of the small molecule relative to the number of $\alpha \mathrm{S}$ monomers in the fibrils. Early experiments with a more limited number of labeled constructs showed that no significant changes in FP occurred with substoichiometric levels of small molecules (see Figure S20).

We have further analyzed the dopamine, EGCG, and NDGA reactions at the $3 \mathrm{~h}$ time point by pelleting the insoluble material and measuring the FP of the resuspended pellet and the supernatant (Figure 4F). We performed the resuspensions with buffer containing the small molecule used in the 
disaggregation reaction to ensure that switching the solvent did not perturb the reversible binding of small molecules to alter the conformations of the pelleted $\alpha \mathrm{S}$ aggregates. We found that the insoluble fractions of the dopamine-treated samples and buffer-treated samples had FP readings that were similar at all positions except 62 . The FP values for the insoluble fractions of the EGCG- and NDGA-treated samples were slightly higher at $\mathrm{N}$-terminal and NAC positions, but lower at the far C-terminus. TEM images of WT $\alpha$ S fibrils treated with the small molecules show fibrils that are not significantly different in morphology than those treated with buffer alone (Figure $4 \mathrm{H}-\mathrm{K}$, additional images in Supporting Information). The most obvious difference is a reduction in the surface coverage of the TEM grids following treatment with EGCG or NDGA. The TEM images are consistent with gel-based measurements of the amount of protein remaining in the insoluble fraction (Figure $4 \mathrm{E}$ and Figure S25A,B).

Examination of the FP values for the soluble fractions shows a clear difference among dopamine, EGCG, and NDGA treatment (Figure 4G). Dopamine samples have FP values that are relatively similar to monomer values at each position, although gel analysis shows that very little protein is solubilized by dopamine treatment as compared to EGCG or NDGA treatment. It is notable that the dopamine FP values for positions 123 and 136 are higher, consistent with reports that dopamine binds to this region of $\alpha \mathrm{S}^{50}$ EGCG FP readings are consistent with the formation of large, amorphous aggregates, as previously postulated. ${ }^{23,47}$ NDGA FP values at each position are also higher than for monomers or micelle-bound $\alpha \mathrm{S}$, except for a very mobile $\mathrm{C}$-terminus. These data demonstrate that both EGCG and NDGA are able to solubilize $\alpha$ S from fibrils and lead to increased degrees of conformational freedom in many regions of the protein, indicating a significant structural change.

We have considered two potential mechanisms for the interpretation of these data. For mechanism 1, it is known that $\alpha \mathrm{S}$ monomers can be shed from fibrils at low levels. ${ }^{15,53}$ Indeed, our own size exclusion chromatography (SEC) analysis demonstrates the shedding of 5-10\% monomer under bufferonly disaggregation control conditions (see Figures S18 and S19). Therefore, it is possible that EGCG or NDGA binds to these shed monomers, leading to the formation of the soluble oligomers observed by FP. This monomer-to-oligomer transition would drive further fibril-to-monomer transitions by mass action and would effectively convert fibrils to oligomers. For mechanism 2, a simpler model for disaggregation would involve direct binding of EGCG or NDGA to fibrils, inducing a conformational change that leads directly to the shedding of oligomers. Of course, hybrid mechanisms are also possible, but for the preliminary studies presented here, we considered only these two mechanistic extremes.

Several lines of evidence support mechanism 2, direct binding of the small molecules to fibrils. First, treating WT fibrils with EGCG or NDGA in the presence of ThT shows a rapid decrease in ThT fluorescence, with nearly complete disappearance after $15 \mathrm{~min}$ (Figure S17). However, SDSPAGE experiments demonstrate that the amounts of insoluble $\alpha \mathrm{S}$ are still large $(60-70 \%)$ after a 15 min EGCG or NDGA treatment (Figure S26). This implies that the decrease in ThT fluorescence is the result of interactions of EGCG and NDGA with fibril-bound ThT or competition for binding sites on the fibrils, rather than actual disaggregation. Second, treatment of the labeled $\alpha \mathrm{S}$ monomer with dopamine, EGCG, or NDGA does not lead to an observed change in FP over the time frame of the experiment, showing that the small molecules do not trigger conformational change or oligomerization that can be observed by FP when added to monomer (Figure S11). Third, we have observed fluorophore quenching shortly after addition of the small molecules to fibrils containing $\alpha S-C^{\text {Fam }}{ }_{X}$ variants, which implies contact between the EGCG or NDGA and each of the Fam labels (Figure S22). Taken together, these results suggest that EGCG and NDGA directly interact with fibrils at multiple sites, causing changes in conformational freedom reported by the fluorescent label.

The observed FP values for the solubilized species imply that EGCG-solubilized $\alpha \mathrm{S}$ aggregates are less conformationally mobile with respect to the fluorophore sites than NDGAsolubilized aggregates (possibly involving aggregation of the small molecules, as well), but additional study will be necessary to confirm this. Preliminary CD studies of the dopamine-, EGCG-, and NDGA-treated fibrils show that substantial $\beta$-sheet character is still present in all three cases (see Figure S27). SEC analysis reveals the presence of oligomers following treatment with EGCG (see Figures S18 and S19). The observed peak intensity of the oligomeric species decreases following sedimentation, indicating limited solubility and/or equilibrium with sufficiently large species that they cannot be observed by SEC. Treatment of fibrils with NDGA does not lead to the observation of an oligomeric $\alpha \mathrm{S}$ peak in the SEC chromatogram, suggesting that the species formed are sufficiently large that they are also filtered out upon loading of the SEC column. It is likely that the solubilized species involve covalent or noncovalent adducts with oxidized forms of EGCG or NDGA. Oxidation reactions of dopamine, ${ }^{54}$ EGCG, ${ }^{55,56}$ and NDGA $^{57,58}$ can lead to complex mixtures of molecules. Oxidized dopamine metabolites have been reported to form covalent adducts with $\alpha \mathrm{S}^{59-61}$ EGCG has been proposed to form covalent or noncovalent adducts with proteins, ${ }^{62}$ including amyloid fibrils. ${ }^{63}$ Our preliminary studies with tetramethylated NDGA (mNDGA) indicate that oxidation may indeed be important to disaggregation, as mNDGA, which cannot form $o$-quinone oxidation products, does not have a substantial effect on $\alpha \mathrm{S}$ fibrils (see Figure S26). Further investigations of the solubilized products using biophysical techniques and treatments with specific oxidation products are underway and will be reported subsequently.

\section{CONCLUSIONS}

Here, we have shown that site-specifically labeled $\alpha \mathrm{S}$ can be used in FP experiments to monitor various processes, including SDS- and LUV-induced folding, fibril formation, disaggregation, and fibril remodeling induced by exogenous small molecules. In all cases, we have shown that site-specific Fam labels yield information about local protein dynamics in a manner that is compatible with screening in multiwell plate formats. Although other groups have shown that certain small molecules can remodel $\alpha \mathrm{S}$ fibrils into amorphous, nontoxic species, the molecular basis for this effect is not well understood and is likely obscured by competition between the small molecule and reporter dyes, such as ThT or CR, for protein binding sites. Our results suggest that EGCG preferentially binds to and remodels the $\mathrm{C}$-terminus of $\alpha \mathrm{S}$ in fibrils, yielding soluble aggregates, whereas excess dopamine has little effect on the structure of $\alpha \mathrm{S}$ fibrils. NDGA, which has not previously been explored as a disaggregant, had the most dramatic effect, mobilizing all regions of $\alpha \mathrm{S}$. Given the evidence of a role for 
oxidation and multiple binding sites in the effects of EGCG and NDGA on fibrils, further study will be needed to fully understand the mechanistic details of their actions and the structures of the resulting solubilized aggregates. Nonetheless, our studies show that our FP assay can yield information about the disaggregation process more accurately than information from typical ThT or CR high-throughput experiments. In the future, FP assays with labeled constructs validated here can be used to screen small molecules in efforts to discover novel compounds with enhanced therapeutic potential for PD.

\section{ASSOCIATED CONTENT}

\section{S Supporting Information}

The Supporting Information is available free of charge on the ACS Publications website at DOI: 10.1021/acs.biochem.6b01060.

Protocols for protein expression, labeling, and characterization as well as additional descriptions of aggregation, micelle and vesicle binding, and small molecule remodeling assays $(\mathrm{PDF})$

\section{AUTHOR INFORMATION}

\section{Corresponding Author}

*E-mail: ejpetersson@sas.upenn.edu.

\section{Present Addresses}

"R.F.W.: Department of Chemistry, Yale University, 225 Prospect St., New Haven, CT 06520.

${ }^{\perp}$ M.C.: Department of Chemistry, Faculty of Science, Bilkent University, Ankara 06800, Turkey.

\section{Author Contributions}

C.M.H., C.L.C., and R.F.W. contributed equally to this work.

\section{Funding}

This work was supported by funding from the University of Pennsylvania, as well as grants from the National Institutes of Health (NS081033 to E.J.P. and GM 097552 to T.B.). R.F.W. thanks the National Institutes of Health (NIH) for previous funding through the Chemistry-Biology Interface Training Program (T32 GM07133). C.M.H. is supported by an NIH Age Related Neurodegenerative Disease Training Grant fellowship (T32AG000255).

\section{Notes}

The authors declare no competing financial interest.

\section{ACKNOWLEDGMENTS}

The National Science Foundation provided support for the MALDI-MS (NSF MRI-0820996) and CD (NSF DMR0520020) instruments. Electron microscopy was performed at the University of Pennsylvania Electron Microscopy Resource Laboratory (EMRL). C.M.H. thanks Dr. Dewight Williams for assistance with TEM.

\section{REFERENCES}

(1) Auluck, P. K., Caraveo, G., and Lindquist, S. (2010) Annu. Rev. Cell Dev. Biol. 26, 211-233.

(2) Bartels, T., Choi, J. G., and Selkoe, D. J. (2011) Nature 477, 107110.

(3) Dettmer, U., Newman, A. J., Soldner, F., Luth, E. S., Kim, N. C., Von Saucken, V. E., Sanderson, J. B., Jaenisch, R., Bartels, T., and Selkoe, D. (2015) Nat. Commun. 6, 7314.

(4) Dettmer, U., Newman, A. J., Von Saucken, V. E., Bartels, T., and Selkoe, D. (2015) Proc. Natl. Acad. Sci. U. S. A. 112, 9596-9601.
(5) Dettmer, U., Selkoe, D., and Bartels, T. (2016) Curr. Opin. Neurobiol. 36, 15-22.

(6) Theillet, F. X., Binolfi, A., Bekei, B., Martorana, A., Rose, H. M., Stuiver, M., Verzini, S., Lorenz, D., Van Rossum, M., Goldfarb, D., and Selenko, P. (2016) Nature 530, 45-50.

(7) Uversky, V. N. (2003) J. Biomol. Struct. Dyn. 21, 211-234.

(8) Chandra, S., Chen, X., Rizo, J., Jahn, R., and Südhof, T. C. (2003) J. Biol. Chem. 278, 15313-15318.

(9) Eliezer, D., Kutluay, E., Bussell, R., Jr., and Browne, G. (2001) J. Mol. Biol. 307, 1061-1073.

(10) Celej, M. S., Sarroukh, R., Goormaghtigh, E., Fidelio, G. D., Ruysschaert, J.-M., and Raussens, V. (2012) Biochem. J. 443, 719-726.

(11) Cremades, N., Cohen, S. A., Deas, E., Abramov, A. Y., Chen, A. Y., Orte, A., Sandal, M., Clarke, R. W., Dunne, P., Aprile, F. A., Bertoncini, C. W., Wood, N. W., Knowles, T. P. J., Dobson, C. M., and Klenerman, D. (2012) Cell 149, 1048-1059.

(12) Dusa, A., Kaylor, J., Edridge, S., Bodner, N., Hong, D.-P., and Fink, A. L. (2006) Biochemistry 45, 2752-2760.

(13) Fink, A. L. (2006) Acc. Chem. Res. 39, 628-634.

(14) Kaylor, J., Bodner, N., Edridge, S., Yamin, G., Hong, D.-P., and Fink, A. L. (2005) J. Mol. Biol. 353, 357-372.

(15) Shvadchak, V. V., Claessens, M. M. a. E., and Subramaniam, V. (2015) J. Phys. Chem. B 119, 1912-1918.

(16) Uversky, V. N., Li, J., and Fink, A. L. (2001) J. Biol. Chem. 276, 10737-10744.

(17) Rodriguez, J. A., Ivanova, M. I., Sawaya, M. R., Cascio, D., Reyes, F. E., Shi, D., Sangwan, S., Guenther, E. L., Johnson, L. M., Zhang, M., Jiang, L., Arbing, M. A., Nannenga, B. L., Hattne, J., Whitelegge, J., Brewster, A. S., Messerschmidt, M., Boutet, B., Sauter, N. K., Gonen, T., and Eisenberg, D. S. (2015) Nature 525, 486-490.

(18) Tuttle, M. D., Comellas, G., Nieuwkoop, A. J., Covell, D. J., Berthold, D. A., Kloepper, K. D., Courtney, J. M., Kim, J. K., Barclay, A. M., Kendall, A., Wan, W., Stubbs, G., Schwieters, C. D., Lee, V. M. Y., George, J. M., and Rienstra, C. M. (2016) Nat. Struct. Mol. Biol. 23, 409-415.

(19) Conway, K. A., Harper, J. D., and Lansbury, P. T. (2000) Biochemistry 39, 2552-2563.

(20) Luk, K. C., Kehm, V., Carroll, J., Zhang, B., O'brien, P., Trojanowski, J. Q., and Lee, V. M. (2012) Science 338, 949-953.

(21) Li, J., Zhu, M., Manning-Bog, A. B., Di Monte, D. A., and Fink, A. L. (2004) FASEB J. 18, 962-964.

(22) Singh, P. K., Kotia, V., Ghosh, D., Mohite, G. M., Kumar, A., and Maji, S. K. (2013) ACS Chem. Neurosci. 4, 393-407.

(23) Bieschke, J., Russ, J., Friedrich, R. P., Ehrnhoefer, D. E., Wobst, H., Neugebauer, K., and Wanker, E. E. (2010) Proc. Natl. Acad. Sci. U. S. A. $107,7710-7715$.

(24) Prabhudesai, S., Sinha, S., Attar, A., Kotagiri, A., Fitzmaurice, A., Lakshmanan, R., Ivanova, M., Loo, J., Klärner, F.-G., Schrader, T., Stahl, M., Bitan, G., and Bronstein, J. (2012) Neurotherapeutics 9, 464476 .

(25) Li, J., Zhu, M., Rajamani, S., Uversky, V. N., and Fink, A. L. (2004) Chem. Biol. 11, 1513-1521.

(26) Gautam, S., Karmakar, S., Bose, A., and Chowdhury, P. K. (2014) Biochemistry 53, 4081-4083.

(27) Caruana, M., Högen, T., Levin, J., Hillmer, A., Giese, A., and Vassallo, N. (2011) FEBS Lett. 585, 1113-1120.

(28) Grelle, G., Otto, A., Lorenz, M., Frank, R. F., Wanker, E. E., and Bieschke, J. (2011) Biochemistry 50, 10624-10636.

(29) Lendel, C., Bertoncini, C. W., Cremades, N., Waudby, C. A., Vendruscolo, M., Dobson, C. M., Schenk, D., Christodoulou, J., and Toth, G. (2009) Biochemistry 48, 8322-8334.

(30) Lakowicz, J. R. (2006) Principles of Fluorescence Spectroscopy, 3rd ed., Springer, New York.

(31) Luk, K. C., Hyde, E. G., Trojanowski, J. Q., and Lee, V. M. Y. (2007) Biochemistry 46, 12522-12529.

(32) Wood, S. J., Wypych, J., Steavenson, S., Louis, J. C., Citron, M., and Biere, A. L. (1999) J. Biol. Chem. 274, 19509-19512.

(33) Tian, A., and Baumgart, T. (2009) Biophys. J. 96, 2676-2688. 
(34) Haney, C. M., Wissner, R. F., Warner, J. B., Wang, Y. X. J., Ferrie, J. J., Covell, D. J., Karpowicz, R. J., Lee, V. M. Y., and Petersson, E. J. (2016) Org. Biomol. Chem. 14, 1584-1592.

(35) Tramier, M., and Coppey-Moisan, M. (2008) in Methods in Cell Biology (Kevin, F. S., Ed.) Vol. 85, pp 395-414, Academic Press, New York.

(36) Eliezer, D., Kutluay, E., Bussell, R., Jr., and Browne, G. (2001) J. Mol. Biol. 307, 1061-1073.

(37) Ulmer, T. S., Bax, A., Cole, N. B., and Nussbaum, R. L. (2005) J. Biol. Chem. 280, 9595-9603.

(38) Moosa, M. M., Ferreon, A. C., and Deniz, A. A. (2015) ChemPhysChem 16, 90-94.

(39) Trexler, A. J., and Rhoades, E. (2009) Biochemistry 48, 23042306.

(40) Mizuno, N., Varkey, J., Kegulian, N. C., Hegde, B. G., Cheng, N. Q., Langen, R., and Steven, A. C. (2012) J. Biol. Chem. 287, 2930129311.

(41) Jao, C. C., Hegde, B. G., Chen, J., Haworth, I. S., and Langen, R. (2008) Proc. Natl. Acad. Sci. U. S. A. 105, 19666-19671.

(42) Bertoncini, C. W., Jung, Y.-S., Fernandez, C. O., Hoyer, W., Griesinger, C., Jovin, T. M., and Zweckstetter, M. (2005) Proc. Natl. Acad. Sci. U. S. A. 102, 1430-1435.

(43) Dedmon, M. M., Lindorff-Larsen, K., Christodoulou, J., Vendruscolo, M., and Dobson, C. M. (2005) J. Am. Chem. Soc. 127, 476-477.

(44) Johansson, L. B. A. (1990) J. Chem. Soc., Faraday Trans. 86, 2103-2107.

(45) Guo, J. L., Covell, D. J., Daniels, J. P., Iba, M., Stieber, A., Zhang, B., Riddle, D. M., Kwong, L. K., Xu, Y., Trojanowski, J. Q., and Lee, V. M. Y. (2013) Cell 154, 103-117.

(46) Conway, K. A., Rochet, J.-C., Bieganski, R. M., and Lansbury, P. T. (2001) Science 294, 1346-1349.

(47) Ehrnhoefer, D. E., Bieschke, J., Boeddrich, A., Herbst, M., Masino, L., Lurz, R., Engemann, S., Pastore, A., and Wanker, E. E. (2008) Nat. Struct. Mol. Biol. 15, 558-566.

(48) Rochet, J.-C., Fleming Outeiro, T., Conway, K., Ding, T., Volles, M., Lashuel, H., Bieganski, R., Lindquist, S., and Lansbury, P. (2004) J. Mol. Neurosci. 23, 023-034.

(49) Lorenzen, N., Nielsen, S. B., Yoshimura, Y., Vad, B. S., Andersen, C. B., Betzer, C., Kaspersen, J. D., Christiansen, G., Pedersen, J. S., Jensen, P. H., Mulder, F. a. A., and Otzen, D. E. (2014) J. Biol. Chem. 289, 21299-21310.

(50) Norris, E. H., Giasson, B. I., Hodara, R., Xu, S., Trojanowski, J. Q., Ischiropoulos, H., and Lee, V. M.-Y. (2005) J. Biol. Chem. 280, 21212-21219.

(51) Rekas, A., Knott, R., Sokolova, A., Barnham, K., Perez, K., Masters, C., Drew, S., Cappai, R., Curtain, C., and Pham, C. L. (2010) Eur. Biophys. J. 39, 1407-1419.

(52) Planchard, M. S., Exley, S. E., Morgan, S. E., and Rangachari, V. (2014) Protein Sci. 23, 1369-1379.

(53) Knowles, T. P. J., Waudby, C. A., Devlin, G. L., Cohen, S. I. A., Aguzzi, A., Vendruscolo, M., Terentjev, E. M., Welland, M. E., and Dobson, C. M. (2009) Science 326, 1533-1537.

(54) Herlinger, E., Jameson, R. F., and Linert, W. (1995) J. Chem. Soc., Perkin Trans. 2, 259-263.

(55) Valcic, S., Burr, J. A., Timmermann, B. N., and Liebler, D. C. (2000) Chem. Res. Toxicol. 13, 801-810.

(56) Mochizuki, M., Yamazaki, S.-I., Kano, K., and Ikeda, T. (2002) Biochim. Biophys. Acta, Gen. Subj. 1569, 35-44.

(57) Billinsky, J. L., and Krol, E. S. (2008) J. Nat. Prod. 71, 16121615.

(58) Asiamah, I., Hodgson, H. L., Maloney, K., Allen, K. J. H., and Krol, E. S. (2015) Bioorg. Med. Chem. 23, 7007-7014.

(59) Follmer, C., Coelho-Cerqueira, E., Yatabe-Franco, D. Y., Araujo, G. D. T., Pinheiro, A. S., Domont, G. B., and Eliezer, D. (2015) J. Biol. Chem. 290, 27660-27679.

(60) Werner-Allen, J. W., Dumond, J. F., Levine, R. L., and Bax, A. (2016) Angew. Chem. 128, 7500-7504.
(61) Bisaglia, M., Mammi, S., and Bubacco, L. (2007) J. Biol. Chem. 282, 15597-15605.

(62) Ishii, T., Mori, T., Tanaka, T., Mizuno, D., Yamaji, R., Kumazawa, S., Nakayama, T., and Akagawa, M. (2008) Free Radical Biol. Med. 45, 1384-1394.

(63) Palhano, F. L., Lee, J., Grimster, N. P., and Kelly, J. W. (2013) J. Am. Chem. Soc. 135, 7503-7510. 